Nouvelles frontières du récit. Au-delà de l'opposition entre factuel et fictionnel

\title{
Le roman graphique et l'Histoire : pour un récit engagé
}

\section{Elisa Bricco}

\section{(2) OpenEdition}

\section{Journals}

Édition électronique

URL : http://journals.openedition.org/narratologie/6864

DOI : 10.4000/narratologie.6864

ISSN : 1765-307X

Éditeur

LIRCES

Référence électronique

Elisa Bricco, « Le roman graphique et l'Histoire : pour un récit engagé », Cahiers de Narratologie [En

ligne], 26 | 2014, mis en ligne le 01 octobre 2014, consulté le 03 mai 2019. URL : http://

journals.openedition.org/narratologie/6864; DOI : 10.4000/narratologie.6864

Ce document a été généré automatiquement le 3 mai 2019.

Article L.111-1 du Code de la propriété intellectuelle. 


\title{
Le roman graphique et l'Histoire : pour un récit engagé
}

\author{
Elisa Bricco
}

1 Le roman graphique, ou graphic novel, est désormais devenu un produit littéraire à part entière depuis une trentaine d'années, et plus précisément depuis que Will Eisner publia en 1978 Un pacte avec Dieu (A Contract With God). Depuis, la pratique de raconter dans les planches des bandes dessinées des histoires achevées, avec des personnages pourvus d'une profondeur psychologique et d'une vie intérieure complexe, tout en maintenant les caractéristiques des genres typiques de la BD avec leur décor, mais adressés à un public adulte, s'est répandue de manière exceptionnelle. Le développement de la narration graphique est si important qu'aujourd'hui, avec l'apparition de ce que l'on a défini « la nouvelle bande dessinée ${ }^{1}$ ", on peut aisément considérer ces œuvres comme des créations littéraires ou des récits de fiction ${ }^{2}$, d'autant plus que la critique, même universitaire, s'intéresse à ces ouvrages.

Depuis les années 90, une autre révolution a eu lieu dans la production de la bande dessinée : il s'agit de l'apparition de la BD de reportage ${ }^{3}$ mêlée au récit autobiographique, avec Maus de Art Spiegelman (1992), qui a été suivi, dans sa pratique d'élucidation et de compte rendu de notre histoire contemporaine et de ses conséquences sur l'histoire personnelle des auteurs par, entre autres, Joe Sacco, Emmanuel Guibert ou Philippe Squarzoni ${ }^{4}$.

3 Si l'on prend en compte ces deux typologies de récit bédéique d'un point de vue narratologique, il est évident qu'elles intègrent et dépassent la dichotomie entre récit fictionnel et récit factuel. Le roman graphique raconte des récits de fiction et la BD de reportage des récits de faits et d'événements qui ont réellement eu lieu. Cela va dans le sens des affirmations d'Alessandro Leiduan dans l'introduction à ce volume ${ }^{5}$, alors qu'il propose d'aller au-delà de la dichotomie entre récit factuel et récit fictionnel même au niveau théorique, puisque dans la pratique elle est bel et bien dépassée. On constate que l'hybridation narrative dans le domaine fictionnel, où le document est de plus en plus présent, et la pratique de formuler des hypothèses dans le domaine du récit factuel relèvent du même acte créatif, et engendrent une porosité entre les deux typologies de 
récit qui en résultent en quelque sorte minées de l'intérieur. Désormais, lorsqu'on s'intéresse au récit contemporain, cette dialectique entre les deux manières de construire le récit ne peut être que constatée et cela s'avère d'autant plus important dans le récit en bande dessinée. En fait, il n'est pas aisé d'établir des frontières entre les aspects modaux du récit dans ce genre de création hybride et composite, intersémiotique, qu'est la BD. Ici, plus encore que dans tout autre ouvrage littéraire et artistique, la plume et le pinceau de l'auteur interviennent dans le choix des éléments à montrer en plus de ceux à raconter, et son point de vue, son appréhension personnelle de la réalité, son imagination, s'intègrent au récit, même dans les bandes dessinées de reportage où le réel apparaît dans sa sincérité la plus concrète.

4 Afin de mettre en lumière ces caractéristiques de la BD contemporaine où le factuel et le fictionnel fusionnent et s'interpénètrent, nous analyserons quatre albums où l'Histoire du $\mathrm{XX}^{\mathrm{e}}$ siècle est affrontée et racontée à des degrés de réalisme différents. Il s'agit d'Un homme est mort de Kris et Etienne Davodeau (2006), de Appunti per una storia di guerra de Gipi (2006), de Coupures irlandaises de Kris \& Vincent Bailly (2008) et des Quaderni ucraini d'Igort (2010). Ce sont des ouvrages qui utilisent les mêmes codes sémiotiques et les mêmes stratégies de construction du récit visuel et textuel pour raconter des événements tragiques de notre Histoire contemporaine: une grève à Brest dans l'après-guerre, la guerre en ex-Yougoslavie, la guerre civile en Irlande du Nord et l'oppression stalinienne en Ukraine pendant les années 30. Ces ouvrages sont de véritables romans graphiques et ils ne peuvent pas être considérés comme de simples bandes dessinées, parce que les codes de construction du récit en images y sont totalement bouleversés et répondent aux exigences créatrices et narratives de l'auteur par rapport à l'histoire qu'il veut raconter. Par-delà le gaufrier traditionnel, les romans graphiques pris en compte dans cette étude jouent avec les images, et les textes sont insérés dans les pages suivant les nécessités de la narration. Par conséquent, il s'agit de récits où l'activité du lecteur est mise à contribution dans la construction de l'histoire et dans son appréhension. En effet, non seulement la BD implique des codes de lecture tout à fait particuliers, par lesquels le lecteur est appelé à interpréter des récits composites où le dessin raconte ce que le texte ne dit pas, où les espaces blancs entres les cases sont des lieux à signification et où la couleur et le style graphique expriment des sentiments et des sensations ${ }^{6}$, mais de plus, ici, le lecteur doit suivre le parcours mental de l'auteur sans lien avec le déroulement chronologique de l'histoire ni la mise en page, qui n'est parfois pas linéaire.

Enfin, il nous semble que ces quatre ouvrages illustrent une tendance de plus en plus ancrée dans la création littéraire aujourd'hui, c'est-à-dire l'engagement de l'auteur dans le monde contemporain, son implication personnelle dans ses récits où le mélange de faits réels et de récit fictionnel ou testimonial porte à une hybridation du factuel par le fictionnel et vice-versa. Ainsi, la confrontation de ces quatre lectures du contemporain nous permettra d'interroger la position auctoriale des créateurs, puisqu'ils dénoncent des situations tragiques, méconnues ou mal connues, et se posent en témoins ou bien en sujets interrogateurs de la réalité historique racontée. En outre, le fait que tous les volumes contiennent des textes liminaires, des paratextes où l'auteur explique la situation qui a engendré la création, ou qu'il insère des textes et articles pour approfondir le sujet proposé, révèle une posture ${ }^{7}$ active par rapport à la réception des ouvrages et une volonté de partager sa réflexion avec le lecteur. Cette posture engagée nous paraît être l'un des traits les plus intéressants de la création artistique aujourd'hui. 


\section{Du factuel au fictionnel : du réalisme à l'affabulation}

6 Nous aborderons les caractéristiques de la narration et de la structure des récits dans les analyses de chaque album. Mais avant de les présenter dans le détail, il est important de préciser que nous les avons choisis parce que ces quatre albums peuvent être situés dans des positions intermédiaires dans une ligne horizontale qui va d'un pôle où le récit est factuel à un pôle fictionnel opposé : cela signifie que le rapport entre factuel et fictionnel y est toujours présent, mais à des degrés différents. Voici la ligne de visualisation qui correspond selon nous au rapport que ces récits entretiennent avec l'imagination et le document ; par la suite, nous illustreront les caractéristiques de cette dialectique ainsi qu'elle se développe dans chaque album :

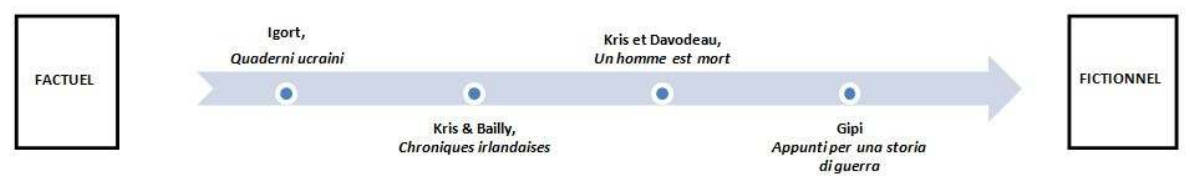

\section{Première étape : du côté du factuel}

7 À l'extrémité du pôle factuel, on situe Les Quaderni ucraini. Mémoires du temps de l'URSS d'Igort. Dans cet album on raconte les conséquences de la grande famine qui a frappé l'Ukraine dans les années 30 et l'exploitation de cette disgrâce par Staline, qui en a profité pour faire face à une résistance à son pouvoir, aboutissant à l'annihilation complète de celle-ci. Cette histoire, presque méconnue, est racontée par un narrateur intradiégétique qui relate un voyage qui a "duré presque deux ans ${ }^{8}$ ». Il s'agit d'un récit documentaire et d'un compte rendu d'enquête, où l'auteur reprend les propos des témoins qu'il a rencontrés au hasard de ses déplacements et qu'il a interrogés et interviewés. " $\mathrm{Al}$ principio l'Ucraina era per me qualcosa di indistinto, una nuvola appartenente al firmamento sovietico. / Poi ho cominciato a frequentarla e i nomi esotici che sentivo in casa sin dall'infanzia: Kiev, Odessa, Poltava, Sebastopoli, Ialta, divennero paesaggi concreti. / Come è stata la vita durante e dopo il comunismo da queste parti? Me lo domandavo sinceramente ${ }^{9}$.»

8 Si nous connaissons imparfaitement la réalité économique et sociale des pays de l'exURSS et leur histoire sous le régime stalinien, toutefois sans doute connait-on moins bien encore les détails du traitement inhumain subi par les populations ukrainiennes à partir des années 30 ; par ailleurs, on oublie trop souvent que ce territoire a été dévasté par la catastrophe de Tchernobyl. Les cahiers d'Igort jettent un regard sur l'Ukraine contemporaine en cherchant les causes qui peuvent en expliquer la situation actuelle par des antécédents historiques. Ainsi, Igort construit son récit pas le biais des témoignages des personnes qu'il a rencontrées en Ukraine et qui peu à peu lui ont raconté leurs petites histoires qui jettent une lumière nouvelles sur la grande Histoire.

Yelena mi racconta la storia di Anya, che, anni fa, era estate, è stata colta di sorpresa da un acquazzone nella città di Energodar, dove c'era una centrale atomica.

Tornata a casa ha fatto una doccia e si è occupata delle faccende domestiche, poi è andata a letto. 
Si è svegliata ed era particolarmente calva. I suoi capelli sul cuscino. I dottori dicono che la roba che era nell'aria dispersa dalla centrale, con l'acqua, ha fatto reazione.

Aveva 30 anni, Anna. Elena non l'ha più vista, non sa se è ancora viva. Le persone di città come Enegodar o Chernobyl hanno divieto di parlare di quello che succede lì.

Questo divieto di sovietiche origini, oggi rimbalza ovunque, per i 4 angoli di quello che fu l'impero comunista. Me ne accorgo presto.

Indipendenza o meno, spesso gli ucraini di questa parte geografica si definiscono "russi". È come se le radici di quella che è stata l'Unione Sovietica, la Sovetsky Soyuz, fossero ancora vive e palpitanti.

Un immenso territorio abitato da usi e convenzioni che sono sopravvissuti, incrostazioni di un passato che non vuole estinguersi.

Basta grattare un poco e sotto la riservatezza sovietica senti scorrere la voglia di essere ascoltati.

Io mi sono trovato lì nei giorni in cui ricorreva il ventennale della caduta del muro di Berlino.

Ho teso l'orecchio ad ascoltare le storie e ho deciso di disegnarle. Semplicemente non ce la facevo a tenermele dentro, neppure io.

Sono storie vere, di persone incontrate casualmente, per strada, cui è toccato in sorte di nascere e vivere stretti nell'abbraccio della cortina di ferro ${ }^{10}$.

9 Cette longue citation est importante puisqu'elle introduit le sujet du volume et l'auteur y établit aussi son pacte de lecture. Il y fournit des informations importantes concernant le contexte et l'occasion de son écriture et il propose son ethos auctorial de manière très explicite. Il est impliqué dans la narration, comme un historien, parce qu'il a recueilli les témoignages de ses narrateurs secondaires, mais il partage avec eux le jugement sévère sur la cruauté de l'Histoire laissant de côté l'objectivité documentaire pour prendre parti : les phrases et les mots qu'il utilise éclaircissent de l'intérieur de l'ouvrage sa posture empathique vis-à-vis des récits qu'il entend et qu'il reproduit : «incrostazioni di un passato che non vuole estinguersi (...) non ce la facevo a tenermele dentro, neppure io $»^{11}$. Les cahiers présentent l'alternance de témoignages et de recherches personnelles de Igort, qui intervient aussi pour approfondir quelques aspects de la vie quotidienne ukrainienne sous le régime soviétique, pour expliquer les procédures administratives auxquelles les citoyens étaient soumis et pour proposer des statistiques sur les événements historiques. Les recherches et les mises au point documentaires de l'auteur contribuent à tisser le fil du récit, tout en accompagnant son regard personnel. Il en résulte un récit fragmentaire, une mosaïque composée de témoignages enregistrés, (1. Storia di Serafina Andreyevna, 4. Storia di Nikolay Vasilievich, 6. Storia di Maria Ivanovna, 8. Storia di Nikolay Ivanovich) et de récits à la première personne de l'auteur (7. La terra, 9. Radiazia), qui sont accompagnés et intégrés par des approfondissements documentaires (2. I kulaki, 3. Litania bolscevica assassina, 5. Conformemente...). Chaque partie concourt ainsi à reconstruire une histoire qui sera naturellement partielle et partiale mais, en même temps aussi, assez complète, vu l'hétérogénéité des narrateurs et des points de vue présentés.

10 Comme nous l'avons expliqué, la place de l'auteur dans l'ouvrage est très visible, même s'il n'apparaît jamais représenté par le dessin, non seulement dans le texte des récitatifs, mais surtout dans la composition et le traitement des images. En effet, dans les parties consacrées aux témoignages et aux souvenirs personnels, les récits sont en couleurs, tandis que les parties avec les approfondissements documentaires sont en noir et blanc et le trait des dessins est beaucoup plus simple - parfois ils sont simplement esquissés. En outre, dans chacune de ces différentes parties, la mise en page est traitée différemment. 
Dans les récits rapportés les planches sont généralement divisées en cinq ou six cases de manière très régulière, dans chacune d'elles une image bandeau est suivie ou entourée d'autres doubles cases. Par contre, les comptes rendus d'Igort ne sont composés que d'images bandeau prenant toute la largeur de la planche, la divisant ainsi en couches horizontales. Le récit y est beaucoup plus métaphorique et le dessin joue un rôle prédominant dans la construction du sens et de la narration. Le rôle du lecteur devient ici très important parce qu'il doit inférer de manière considérable, lire entre les images et composer le sens du récit. Enfin, les planches consacrées aux documents ne présentent pas de gaufrier: ce sont des images qui remplissent les pages en entier sans indiquer un parcours de lecture, mais seulement en exposant des informations directement, parfois sans commentaire. En conclusion, vu le traitement graphique différent des typologies de récit, technique qui explicite le fait que l'auteur compose un récit hétérogène, à partir de témoignages et de documents authentiques qu'il reproduit sans interférer avec des commentaires, nous pouvons situer cet ouvrage dans la partie la plus proche du factuel dans la ligne ci-dessus. Il s'agit en tout cas d'un ouvrage artistique où la part de création personnelle est assez grande et très aisément repérable.

\section{Deuxième étape : où le souvenir s'imprime sur la réalité}

11 Le deuxième ouvrage de notre corpus est une BD de fiction, documentaire et en même temps autobiographique, qui s'intitule Coupures irlandaises ${ }^{12}$, et a été écrite par Kris tandis que Vincent Bailly s'est occupé de la réalisation des dessins. Dans notre ligne du factuel au fictionnel, cet album se situe dans une position intermédiaire mais encore proche du pôle factuel, puisqu'il s'agit d'un récit autobiographique où Kris se met en scène et raconte une aventure qu'il a vécue pendant son adolescence. Or, son histoire personnelle a croisé la grande Histoire lors d'un voyage en Irlande du nord, à Belfast dans l'été 1987. Le rapport factuel-fictionnel et le souci de la documentation de la part de l'auteur est mis en évidence dès la première planche lorsqu'il explique, par l'intermédiaire de son héros : "J'ai toujours voulu raconter des histoires. / Enfant, je m'en étais fait la promesse. / Je collectionnais les coupures de journaux sur les sujets qui m'intéressaient. / Pour plus tard. / Pour quand je serais grand. » [3] Voilà aussi que le titre trouve son explication et que le rapport entre autobiographie et documentation est éclairé : nous comprenons aisément que le récit que nous lirons est un texte qui se situe à l'intersection des deux modalités du discours. Et en fait, en poursuivant la lecture, nous plongeons dans l'atmosphère un peu sombre et pas tout à fait compréhensible de l'Irlande du Nord des années 70 et 80 , alors que catholiques et protestants étaient engagés dans une lutte fratricide. L'apport de cet ouvrage à la compréhension de la triste et sombre atmosphère de l'époque est essentiel, parce qu'elle est racontée par quelqu'un qui l'a vécue personnellement. En outre, l'album comprend aussi des planches documentaires qui rendent compte de la réalité irlandaise de l'époque, et un dossier final, En guise de cicatrice , qui scelle définitivement l'album avec des mises au point, des articles de journaux et des approfondissements sur Le contexte historique, avec les témoignages de quelques protagonistes de cette époque.

12 Dans cet ouvrage tous les moyens d'expression de la BD sont mis à contribution afin de créer un tout unique où le dessin, la composition des planches, la narration et le texte concourent à nous faire comprendre cette réalité si difficile à expliquer. Un narrateur 
interne, le jeune Christophe, relate son aventure personnelle en intervenant directement dans les récitatifs qui alternent avec les reconstructions des dialogues. Chaque planche propose un rythme différent et s'adapte encore une fois aux exigences du récit. Par exemple, lorsque Christophe et son copain Nicolas fêtent leur voyage prochain en Irlande, dans une page au rythme très serré ${ }^{13}$ où chacun des quatre bandeaux horizontaux est coupé différemment pour donner justement du mouvement et où la focalisation accompagne une sorte de caméra imaginaire qui tourne dans leur chambre: on se rapproche et s'éloigne d'eux, on les suit dans leurs mouvements selon des angles différents. Dans d'autres moments du récit le rythme ralentit: par exemple lors de leur voyage vers l'Irlande, dans une planche qui se développe dans le sens horizontal [11]. Ailleurs, l'ennui des deux jeunes français, qui sont obligés de rester et de ne pas s'éloigner du quartier catholique après un attentat, est représenté par la lenteur dans des développements verticaux. Par conséquent, l'œil du lecteur, au lieu de faire un parcours de gauche à droite et de haut en bas comme pour une lecture linéaire habituelle, se déplace ici de haut en bas à chaque case [46] obligeant le lecteur à un exercice assez inusuel : l'œil va et vient de haut en bas et, à chaque passage vers la droite, est obligé de recommencer le même mouvement basculant en vertical.

Ici, les couleurs aussi jouent un rôle important, puisqu'elles contribuent à créer les atmosphères: la maison de la famille Nevlin qui accueille Nicolas dans le quartier des Markets à Belfast, habité par les catholiques, est caractérisée par les couleurs chaudes [20] qui représentent l'intimité de la famille, l'accueil chaleureux et ouverture d'esprit démontré avec les deux jeunes français. Par contre, la famille Nicholl, protestante qui habite dans les quartiers nord de la ville, a une maison qui est connotée par des couleurs plus froides, le vert, très irlandais, le bleu et le gris [25], ce qui représente en quelque sorte le contraste entre les deux situations sociales et humaines. Parfois, la couleur joue un rôle important aussi dans la mise en place d'une atmosphère: lors d'une nuit de combats urbains, le jaune remplit les pages [54-62] en contribuant à rendre compte de l'utilisation de la lumière artificielle pendant la nuit, mais aussi en insinuant un malaise chez le lecteur, le jaune étant la couleur de la haine, et ici c'est une haine presque raciale, civile, qui est en jeu.

Les dessins de Bailly, bien que très proches des esquisses et donc peu définis, parviennent en tout cas à bien représenter les détails des scènes, les mimiques des personnages et à rendre compte des enjeux des relations interpersonnelles. Comme on l'a écrit, la construction du récit est très vraisemblable, et surtout on arrive à bien percevoir le climat de militarisation de cette région à cette époque-là par la présence de barrages barbelés en ville [42]; par la présence des soldats armés dans les rues, [31] des tanks et des hélicoptères, des raids des soldats dans les maisons civiles, [50] avec aussi, à côté, la présence des militants de l'IRA [44]. C'est toute une situation assez folle que les deux jeunes français ont côtoyée, subie même, et qui a laissé une trace indélébile dans leur esprit, ce qui a fait qu'après vingt ans l'un d'eux a trouvé le courage de raconter cette histoire absurde.

\section{Troisième étape : du factuel vers le fictionnel}

Le troisième album du corpus, Un homme est mort de Kris et Etienne Davodeau ${ }^{14}$, raconte l'histoire d'une grève qui a eu lieu à Brest pendant la reconstruction de l'après-guerre. Il s'agit de la reprise en bande dessinée d'un événement proche du fait divers : le 17 mars 
1950, pendant une manifestation de grévistes, des ouvriers du bâtiment et des chantiers navals, la police a ouvert le feu sur les manifestants en tuant un affilié de la CGT, Édouard Mazé, qui se trouvait au premier rang. Cet ouvrage est le fruit de la collaboration entre deux auteurs : Kris, qui a longtemps réfléchi à la transposition de cette histoire qu'il avait entendue lorsqu'il était adolescent, et Étienne Davodeau, dont la production personnelle était déjà très ancrée dans la réalité avec la publication d'autres $\mathrm{BD}$ documentaires ${ }^{15}$. Les auteurs se sont beaucoup documentés avant d'écrire le scénario de cet album : ils ont fait des recherches d'archive et ils ont rencontré quelques-uns des témoins de l'incident, notamment le réalisateur d'un film documentaire qui avait été transmis lors des rencontres syndicales successives à l'incident. L'album est composé du récit de l'événement tragique et de la création du film documentaire de René Vautrier cité, accompagné d'un dossier final contenant plusieurs documents. Les textes qui clôturent l'album rendent compte de la création du film, de la reconstruction historique de la période de l'après-guerre (Brest. Un chantier ensanglanté de Pierre le Goïc) et sont accompagnés de coupures de journaux d'époque. On y trouve aussi un portrait du cinéaste qui est le véritable protagoniste de ce récit (René Vautrier, tout simplement...) ; un récit de Kris sur Le parcours d'un livre, où il raconte la genèse du projet et la mise en place de celui-ci, jusqu'à sa publication. On a recueilli aussi le témoignage de l'un des participants à la grève, Pierre Cauzien, qui a été blessé dans la même manifestation. Le dossier se ferme sur quelques esquisses et dessins préparatoires de Davodeau.

Ainsi, l'album propose la mise en bande dessinée d'un événement historique encore une fois assez méconnu, un récit factuel qui est raconté d'après une enquête et avec l'aide d'une documentation assez précise et large (coupures de journaux et comptes rendus de témoignages), où l'apport créateur des auteurs est très évident. Et c'est aussi l'histoire d'un film documentaire de propagande, qui a été tourné par une jeune cinéaste de 23 ans, René Vautrier, au lendemain de l'accident et qui a été projeté pendant la grève, au soir, sur les chantiers où se réunissaient les grévistes. Les conditions de la création et de la projection du film étaient si mauvaises qu'après quelques mois la pellicule a été très gravement endommagée. Le point de vue adopté par les deux scénaristes pour raconter ces événements est donc la volonté de retracer l'aventure personnelle de Vautrier, qui est un personnage extérieur à la grève pouvant ainsi poser un regard objectif et désengagé sur la ville de Brest et sur la situation malheureuse des grévistes.

Du point de vue de l'art de la BD, cet album peut être considéré comme un chef d'œuvre, puisqu'il propose la vision très personnelle des événements par les choix graphiques : de la mise en page à la construction du récit planche par planche; du dessin assez symbolique où la reconstruction du passé reste très évocatrice plutôt que fidèle, à l'utilisation de la couleur sépia qui rend parfaitement la représentation du passé16. Comme chez Igort et dans Coupures Irlandaises, ici aussi le gaufrier est plié aux exigences de la représentation : il n'y a pas une planche identique à une autre parce qu'elles sont fonctionnelles au récit et s'adaptent à la matière à raconter. On trouve, par exemple, une planche qui représente la ville en reconstruction, divisée en deux grandes parties où le panorama en bas est annoncé par les trois cases de la partie supérieure où le jeune cinéaste, accompagné de deux affiliés à la CGT, regarde du haut d'un immeuble en construction et ensuite cherche à se repérer dans le nouvel aménagement de Brest. De fil en aiguille, il découvre la nouvelle ville et le lecteur avec lui, en suivant son parcours visuel, d'abord en vision de front et en position de spectateur et ensuite en vision avec ${ }^{17} \mathrm{le}$ personnage même. La ville n'est pas représentée de manière détaillée, mais esquissée. Le 
même basculement de la vision par rapport à la focalisation avec la multiplication des focales se retrouve dans une autre image, où l'on passe d'une vision d'ensemble de la foule des participants aux funérailles d'Édouard Mazé, vue de derrière, à la vision plus rapprochée, de front, d'une partie du cortège et de quelques petites scènes liminaires. Les deux dernières cases de la planche, que l'on pourrait définir des méta-cases puisqu'elles nous illustrent la technique utilisée plus haut, montrent le cinéaste, sujet focalisateur, d'en haut et de front en train de filmer la scène. Ces mouvements de la focalisation, qui miment évidemment ceux du cinéaste, donnent beaucoup de mouvement aux scènes représentées en les rendant très vives ${ }^{18}$.

Deux autres planches qui racontent une projection sont construites avec une symétrie presque parfaite, selon le schéma suivant ${ }^{19}$ :

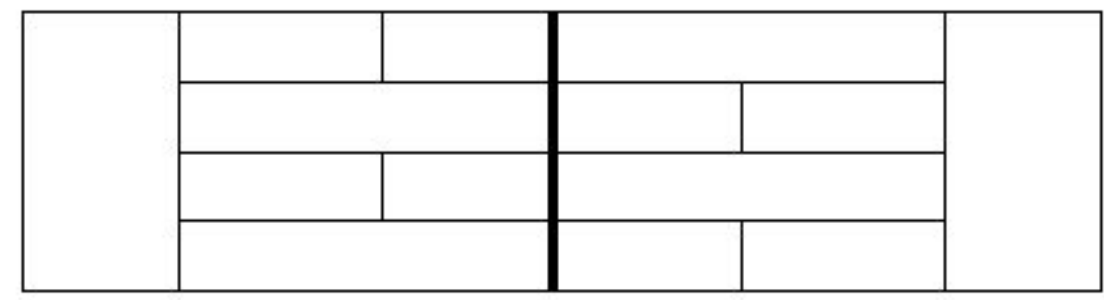

où les deux bandeaux latéraux représentent la projection vue de deux côtés différents, de front et de l'arrière, et les cases intermédiaires montrent des zooms sur le public, pris de différentes positions par rapport à la scène. Cette structure si parcellisé contribue à donner une vue d'ensemble très efficace. D'autres planches montrent différentes projections ${ }^{20}$, et il est intéressant de découvrir la stratégie par laquelle le récit accélère pour rendre compte de la continuité des projections dans le temps : la double planche devient un échiquier où les cases blanches aux récitatifs alternent avec les dessins. Le récit est très scandé et le dessinateur a joué avec la possibilité significative des blancs de la $\mathrm{BD}$, gouffre de vide qui peut ouvrit les portes à l'imagination du lecteur. Enfin, le dernier exemple est encore une double page où les cases ont disparu et il ne reste que quatre bandeaux superposés ${ }^{21}$. Il s'agit d'une énième projection du film lors de laquelle les protagonistes s'aperçoivent que la bande son qu'ils avaient enregistrée, avec la lecture d'un poème de Paul Eluard, «Gabriel Périi ${ }^{22}$ » qu'ils avaient adapté avec le nom d'Edouard Mazé, était détruite et qu'il aurait fallu lire le poème à haute voix à chaque nouvelle projection. L'un des accompagnateurs du cinéaste propose alors de réciter le poème et son élan ainsi que son émotion transparaissent dans la séquence des cases posées horizontalement. Les mouvements du personnage, ses gestes, ses mots qui deviennent des cris sont représentés par les caractères gras très gros, qui se rapetissent ensuite dans la page de droite, où le public a disparu et le personnage reste seul au milieu de l'image. Ses mots sont accompagnés par les mouvements de la focalisation qui comme une caméra bouge de droite à gauche, fragmentant la vision de la scène comme si c'était un caléidoscope. Le dernier bandeau, muet, montre l'homme de dos, épuisé par l'effort et par la douleur qu'il éprouve.

Ainsi se réalise le passage ou bien l'hybridation du factuel par le fictionnel : le lecteur est accompagné à la découverte d'un événement historique dont on lui propose une reconstitution fidèle qui, grâce aux compétences créatrices des deux auteurs, devient plus allusive et métaphorique que réaliste. C'est par la construction du récit et par la 
composition des planches qu'on est induit à imaginer, qu'on comprend les enjeux et le pathos de cette histoire. Nous la percevons de l'intérieur, par la mise en relief des émotions et des sentiments des protagonistes qui nous sont bien représentés par les images.

\section{Quatrième étape : le fictionnel au-delà du factuel}

Le dernier ouvrage du corpus, Appunti per una storia di guerra de Gipi ${ }^{23}$, se situe au pôle à droite de la ligne ci-dessous, celui de la fiction. Dans ce récit on ne trouve aucun élément utile pour indiquer au lecteur qu'il s'agit d'une histoire qui a réellement eu lieu, aucun document ni témoignage ne complète le récit, qui est plus évocateur que réaliste. Gipi a voulu, en fait, produire une métaphore de la guerre, raconter toute guerre par le biais de l'histoire de ses jeunes protagonistes, par laquelle on reconnaît très bien des situations et des lieux évoquant la guerre en ex-Yougoslavie. C'est l'auteur même qui explique sa démarche, son souci de raconter une histoire de guerre qui soit paradigmatique de toute situation de conflit, avec son absurdité foncière, ses abus, ses malentendus et ses injustices :

\footnotetext{
La cosa soprattutto che m'incuriosiva, era il modo in cui tutti noi, io in testa, continuavamo a farci gli affari nostri.

Con pochissimi cambiamenti, forse nessuno, e solo qualche angoscia che radicava nei cuori più sensibili.

Era strana questa cosa ma era successa già mille volte in passato.

Quella guerra, come la guerra in Bosnia, quelle in Africa, in Algeria, in Medio

Oriente, nel Sud Est Asiatico, non erano le "nostre" guerre.

Questo era (è, in realtà) un fatto assodato.

La guerra nella ex-Jugoslavia, per tornare vicini, per esempio, non era la nostra guerra. Era la loro. Era la guerra dei serbi, dei bosniaci, dei croati. [p. $127]^{24}$
}

Le récit est raconté par l'un des protagonistes, Giuliano, qui rôde avec ses copains Christian et Stefano, dit il killerino, dans la banlieue d'une ville désormais en ruines, à la recherche de nourriture mais aussi d'aventures. La rencontre avec un groupe de miliciens, mi-terroristes mi-voyous, les plonge dans la réalité de la guerre comme elle se déroule à l'arrière : où l'on combat pour survivre, où il faut se débrouiller, et où il est nécessaire de commettre des crimes si on a la prétention d'être le maître de son existence. Les trois jeunes décident de s'affilier à la bande, et l'album rend compte de leur montée dans l'échelle de la délinquance et en même temps leur prise de conscience des règles de cette société marginale où se joue leur initiation, dans une réalité cruelle qui n'épargne pas ces adolescents des horreurs des combats et des actions militaires et criminelles désordonnées et inhumaines. Selon leur capacité de supporter la vue et la mise en place d'actes abjects, ils deviennent de vrais combattants. Ainsi, Stefano, dit $i l$ killerino, le plus cruel des trois, devient aussitôt le chef reconnu de leur petite bande : les criminels qui l'ont engagé lui apprennent la cruauté et le respect de la loi du talion, et le chargent de la responsabilité de petites missions de cette pseudo guerre d'entre les pauvres qui se déroule en marge de la vraie guerre. Après quelques aventures très peu héroïques, au bout de ce voyage en enfer, la bande des malfaiteurs s'engage dans une voie qui devient de plus en plus dangereuse. Giuliano, hésitant, reste en marge et à la fin décide de laisser tomber : il s'enfuit et, resté sans ses compagnons, il doit se confronter à nouveau à la réalité de la paix. Seulement lui, le narrateur, qui avait toujours été à côté 
des autres et ne s'était jamais intégré vraiment dans le groupe ni ne s'était senti tout à fait désinvolte par rapport au crime et à la cruauté, réussit à s'éloigner de cette situation et ensuite à raconter son expérience dans un documentaire. L'album, on le découvre seulement à la fin, est en fait le récit de ses expériences qu'il fait à un journaliste documentariste qui veut faire un film, ce sont des "Notes pour une histoire de guerre " qui sera filmée ensuite.

Dans cet ouvrage, Gipi a voulu donner un exemple, montrant combien la réalité de la guerre nous est très proche, au point de nous toucher un jour. Il a écrit un récit fictionnel où le factuel peut être perçu par l'inférence, dans ce que nous pouvons supposer du réel à travers l'histoire racontée et les moyens mis en place pour la représenter par les images. Pour rendre compte de cette situation de guerre, l'auteur a choisi d'employer l'aquarelle en noir et blanc, de sorte que la grisaille domine la scène. Dans tout ce gris, parfois l'auteur introduit des cases où le dessin est composé par le seul trait du pinceau [12-86], cela lui permet d'intervenir dans la narration pour introduire par exemple les analepses, les moments où surgit le souvenir du killerino, le chef de la petite bande qui a vécu une enfance très difficile marquée par le suicide de son père; mais aussi les rêves ou les cauchemars de Giuliano lorsqu'ils surgissent [17]. Ce sont des situations où l'auteur veut mettre à jour l'émergence de l'inconscient, il veut montrer qu'en dessous d'une apparente assurance, les jeunes gardent les faiblesses de leur âge. En fait, les souvenirs concernent la famille qu'ils ont quittée, c'est-à-dire la vie avant la guerre, et leurs rêves représentent leurs craintes et les expériences dures et difficiles qu'ils doivent affronter [89]. En outre, chez Gipi aussi, on remarque un usage fonctionnel de la planche, l'aménagement des espaces est mis au service de la narration, avec l'alternance de planches descriptives avec des bandeaux horizontaux et d'autres où les cases de grandeurs différentes s'alternent pour suivre le rythme des actions [55-56].

\section{Conclusion}

Pour conclure, il est vrai que la fiction vient parfois intégrer et remplir les trous des récits documentaires, d'histoires ou d'enquêtes, là où les faits n'aident pas assez dans la reconstruction objective et où il est nécessaire de formuler des hypothèses. Et il est vrai également que le récit fictionnel se nourrit de plus en plus de l'enquête documentaire pour se rapprocher de la vérité dans la narration. Gipi, au bout du parcours qui nous a amenés du factuel au fictionnel, démontre comment la fiction vise à rendre compte de la réalité, comment elle parvient à la mettre en scène et aboutit, par le biais de l'imaginaire, à se rapprocher de la vérité objective. C'est une vérité dérivant de la connaissance des êtres humains et de l'expérience personnelle de l'auteur et collective des lecteurs. Cette vérité, que chacun connaît mais que sans doute peu ont le courage de dire explicitement, est rendue encore plus efficace et prégnante chez l'auteur italien par son apparence hors du réel purement documentaire.

Ainsi que nous l'avons annoncé dans l'introduction, tous les ouvrages pris en compte dans cette étude visent à rendre compte et à éclairer des aspects de l'histoire contemporaine qui sont méconnus, ou qui n'ont pas reçu la juste attention par les historiens et par les chroniques. Par le biais des documents et des enquêtes, des témoignages et des recherches personnelles, les auteurs ont réussi à doser le factuel et le fictionnel à des degrés différents, pour créer des ouvrages où l'approche ludique de la 
lecture et de la création de la bande dessinée interagit avec l'aspect cognitif, avec la réflexion qu'implique toute dénonciation et tout récit d'événements tragiques.

\section{NOTES}

1. Hugues Dayez, La Nouvelle Bande Dessinée. Entretiens avec Hugues Dayez. Blain/Blutch/David

B./de Crécy/Dupuis-Berberian/Guibert/Rabaté/ Sfar, Niffle, 2002.

2. Jan Baetens, "Littérature et bande dessinée. Enjeux et limites ", Cahiers de Narratologie, 16 (2009), « Images et récits ». URL : http://narratologie.revues.org/974 Consulté le 04.11.2013

3. L'article de Catherine Saouter fait le point sur cette typologie de création en BD et inclut dans le corpus quelques ouvrages qui seront objet d'analyse ici. "Guerre et BD reportage : entre dialogue et contemplation ", in Expériences de la guerre entre littérature et image, Textes de l'atelier de recherche du 11 et 12.11.2010, Groupe de recherche Intermédialités du témoignage et mémoire $\mathrm{du} \mathrm{XX}^{\mathrm{e}}$ siècle, Université $\mathrm{du}$ Québec à Montréal, URL: http:// experiencedelaguerreecritureimage.uqam.ca/textes/Catherine\%20Saouter_Guerre\%20et\% 20BDreportage\%20entre\%20dialogue\%20et\%20contemplation.pdf Consulté le 09.12.2013.

4. Cf. Pascal Lefevre, Gert Meesters, «Aperçu général de la bande dessinée francophone actuelle », Relief, 2 (3), 2008, p. 290-308. URL: http://www.revue-relief.org/index.php/relief/ article/view/229/322 Consulté le 31.05.2014.

5. Alessandro Leiduan, « Nouvelles frontières du récit. Au-delà de l'opposition entre factuel et fictionnel », Préface au présent numéro 26 des Cahiers de narratologie.

6. Cf. les analyses, entre autres, de Benoît Peeters et Thierry Groensteen.

7. Jérôme Meizoz, Postures littéraires. Mises en scène modernes de l'auteur, Genève, Slatkine, 2007.

8. Igort, Quaderni ucraini. Memorie dei tempi dell'URSS, Milano Mondadori, «Le Strade blu », 2010, les pages ne sont pas numérotées.

9. « Au début l'Ukraine était pour moi quelque chose d'indistinct, un nuage appartenant au firmament soviétique. / Puis j'ai commencé à la fréquenter et les noms exotiques et que j'entendais à la maison depuis l'enfance : Kiev, Odessa, Poltava, Sébastopol, Ialta devinrent des paysages concrets. / Comment était la vie pendant et après le communisme par là ? Je me le demandais sincèrement » (notre traduction). Pour voir quelques images : http://www.igort.com/books-quaderni-ucraini.html

10. «Yelena me raconte l'histoire d'Anya, qui, il y a quelques années, en été, a été prise par surprise par une averse dans la ville d'Energodar, où il y avait une centrale nucléaire.

Rentrée chez elle, elle a pris une douche et s'est occupée du ménage, puis s'est couchée.

Elle s'est réveillée et était très chauve. Les cheveux sur l'oreiller. Les médecins disent que la substance qui était dans l'air dispersé par la centrale, avec l'eau, a produit la réaction.

Elle avait 30 ans, Anna. Elena ne l'a plus vue, elle ne sait pas si elle est encore en vie. Les habitants des villes d'Enegodar ou de Tchernobyl ont interdiction de parler de ce qui se passe là-bas.

Cette interdiction, aux senteurs soviétiques, rebondit désormais partout, aux quatre coins de ce qui fut l'empire communiste. Je le remarque bientôt. Indépendance ou pas, souvent les Ukrainiens de cette zone géographique s'appellent eux-mêmes «Russes». C'est comme 
si les racines de ce qui était l'Union soviétique, la Sovetskii Soyouz, étaient encore vivantes et palpitantes.

Un vaste territoire habité par des coutumes et conventions qui ont survécu, l'incrustation d'un passé qui ne veut pas disparaître.

Il suffit de gratter un peu et on sent glisser sous le secret soviétique le désir d'être entendu. J'étais là-bas quand on a célébré le vingtième anniversaire de la chute du mur de Berlin. J'ai écouté attentivement pour entendre les histoires et j'ai décidé de les dessiner. Tout simplement, je ne pouvais pas supporter de les garder pour moi, moi non plus.

Ce sont des histoires véritables de personnes rencontrées par hasard dans la rue, qui ont eu le sort de naître et de vivre enfermés dans l'étreinte du rideau de fer" (notre traduction).

11. « incrustations d'un passé qui ne veut pas mourir (...) je ne pouvais pas les garder en moimême, moi non plus » (notre traduction).

12. Kris et Vincent Bailly, Coupures irlandaises, Paris, Futuropolis, 2008.

13. Voir l'image de la page $7:$ http://www.bdselection.com/imagesbd/c/coupures-irlandaises/ coupures-irlandaises_1.jpg

14. Kris et Étienne Davodeau, Un homme est mort, Paris, Futuropolis, 2006.

15. Étienne Davodeau, Rural!, Paris, Delcourt, coll. «Encrages », 2001 ; Les Mauvaises Gens : une histoire de militants, Delcourt, 2005.

16. Pour en voir un exemple : http://fr.globedia.com/images/nouvelles/2011/8/16/homme-estmort-kris-etienne-davodeau_3_542409.jpg

17. François Jost, L'œil caméra, Lyon, Presses Universitaires, 1989 et François Jost et André Gaudreault, Le Récit cinématografique, Paris, Nathan, 2000.

18. Voir un exemple :

http://static.skynetblogs.be/media/65133/

dyn002_original_295_418_jpeg_2544156_d41862488068d81ef72c76f613e4706e.jpg

19. Voir p. 46-47.

20. Voir un exemple : http://juvaquatre.free.fr/bd/HMpage44.jpg

21. Voir p. 56-57.

22. Paul Eluard, Au rendez-vous allemand, Paris, Gallimard, 1944.

23. Gipi, Appunti per una storia di guerra, Milano, Rizzoli, 2006.

24. « La chose qui m'a particulièrement intrigué était la façon dont chacun d'entre nous, moi en premier, avons continué à nous occuper de nos affaires. Avec très peu de changements, sans doute aucun, et seulement une certaine angoisse qui s'enracinait dans les cœurs des plus sensibles. C'était étrange, mais cette chose était arrivée mille fois de par le passé. Cette guerre, comme la guerre en Bosnie, celles en Afrique, en Algérie, au Moyen-Orient, en dans le Sud-Est asiatique, ce n'était pas «nos » guerres. Cela était (est, en fait) un fait irréversible. La guerre en ex-Yougoslavie, pour nous rapprocher, par exemple, ce n'était pas notre guerre. C'était la leur. C'était la guerre des Serbes, des Bosniaques, des Croates» (notre traduction). 


\section{RÉSUMÉS}

On a pu assister ces dix dernières années au développement de la bande dessinée de reportage avec un fort ancrage autobiographique. L'analyse de la dialectique qui se met en place entre le récit factuel et le fictionnel dans quatre albums récents permet de vérifier l'hypothèse que les frontières entre ces typologies de discours sont désormais assez poreuses, et que factuel et fictionnel cohabitent, à des degrés d'intensité différents, dans la production bédéique contemporaine, et notamment dans Un homme est mort de Kris et Etienne Davodeau (2006), Appunti per una storia di guerra de Gipi (2006), Coupures irlandaises de Kris \& Vincent Bailly (2008) et Quaderni ucraini d'Igort (2010).

Nel corso degli ultimi dieci anni, si è assistito allo sviluppo di un genere fumettistico di orientamento giornalistico (reportage) e dai forti risvolti autobiografici. L'analisi della dialettica, osservabile in quattro recenti album, tra la dimensione fattuale e la dimensione della finzione conferma l'ipotesi secondo la quale la frontiera tra queste due tipologie di discorso sarebbe ormai porosa al punto da rendere possibile la loro coabitazione, attraverso forme e gradi d'intensità diversi, nella produzione fumettistica contemporanea e, in particolare, in: Un homme est mort di Kris et Etienne Davodeau (2006), Appunti per una storia di guerra di Gipi (2006), Coupures irlandaises di Kris \& Vincent Bailly (2008) e Quaderni ucraini d'Igort (2010).

\section{INDEX}

Mots-clés : bande dessinée, fiction, factuel, Gipi, Kris, Etienne Davodeau, Vincent Bailly, Igort Index chronologique : XXIe siècle

\section{AUTEUR}

\section{ELISA BRICCO}

Université de Gênes 